\title{
Percutaneous Implantation of Venus P-Valve and Melody Valve in a Patient with Dysfunctional Native and Artificial Right Ventricular Outflow Tracts
}

\author{
Basil (Vasileios) D. Thanopoulos, MD, PhD ${ }^{1 *}$, Petros Dardas, MD ${ }^{1}$, Alexandros Kallifatidis, MD ${ }^{1}$, \\ Shakeel A. Quireshi, MBChB, FRCP2 \\ ${ }^{1}$ Interventional Cardiology, St.Luke's Hospital, Thessaloniki, Greece \\ ${ }^{2}$ Department of Paediatric Cardiology, Evelina Children's Hospital, London, United Kingdom
}

\begin{abstract}
Percutaneous pulmonary valve implantation is been increasingly performed for restoring pulmonary competence in appropriately selected patients. However, The currently available valves are not capable of being safely implanted in the majority of patient with outflow tract dysfunction as they cannot accommodate the larger outflow tract diameters seen in most patients. The Venus $P$-valve is a new percutaneous pulmonary valve designed specifically for implantation in large (up to $32 \mathrm{~mm}$ diameters) native right ventricular outflow tracts. In this report we describe the combined use of a Venus P-valve and Melody transcatheter pulmonary valves in a patient with a dysfunctional native and artificial right ventricular outflow tracts.
\end{abstract}

Copyright @ 2019 Science International Corp.

\section{Key Words}

Percutaneous pulmonary valve implantation • Congenital heart disease • Tetralogy of Fallot

\section{Introduction}

Percutaneous pulmonary valve implantation using the Melody ${ }^{\circledR}$ (Medtronic, Minneapolis, MN, USA) (CE Marked 2006) and the Edwards Sapien XT (Edwards

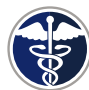

Fax +1 2037853346

E-Mail: jshd@scienceinternational.org

http://structuralheartdisease.org/

\author{
(c) 2019 Journal of Structural Heart Disease \\ Published by Science International Corp. \\ ISSN 2326-4004 \\ Accessible online at: \\ http://structuralheartdisease.org/
}

Lifesciences, Irvine, CA, USA, CE Marked 2017) valves is currently a widely accepted alternative to surgery for the management of selected patients with dysfunctional right ventricular outflow tract conduits or homografts [1]. However, only $<20 \%$ of patients following tetralogy Fallot repair are candidates for these two valves as they cannot be used in large patched right ventricular outflow tracts (RVOTs) [1]. The Venus P-valve (Venus Medtech, Shanghai, China) is a new self-expanding tri-leaflet porcine pericardial tissue percutaneous pulmonary valve designed specifically for implantation in large (up to $32 \mathrm{~mm}$ diameters) native RVOT [2-4]. Early clinical experience with implantations in humans has shown its safety and satisfactory early results. [2-4] In this short communication, we report the first-in-man use of Venus P-valve and Melody $^{\circledR}$ transcatheter valves on separate occasions in a patient with dysfunctional native and artificial RVOT.

\section{Case presentation}

A 21 years male with tetralogy of Fallot underwent surgical correction at the age of 24 months. Due to the presence of an anomalous course of anterior descending artery across the RVOT an initial attempt was made to relieve the sub-pulmonary obstruction

* Corresponding Author:

Basil (Vasileios) D. Thanopoulos, MD, PhD

Interventional Cardiology

St. Luke's Hospital

Panorama 552 36, Thessaloniki, Greece

Tel. +30 210617 9839; Fax: +30 210617 9839; E-Mail: vthanop@otenet.gr 

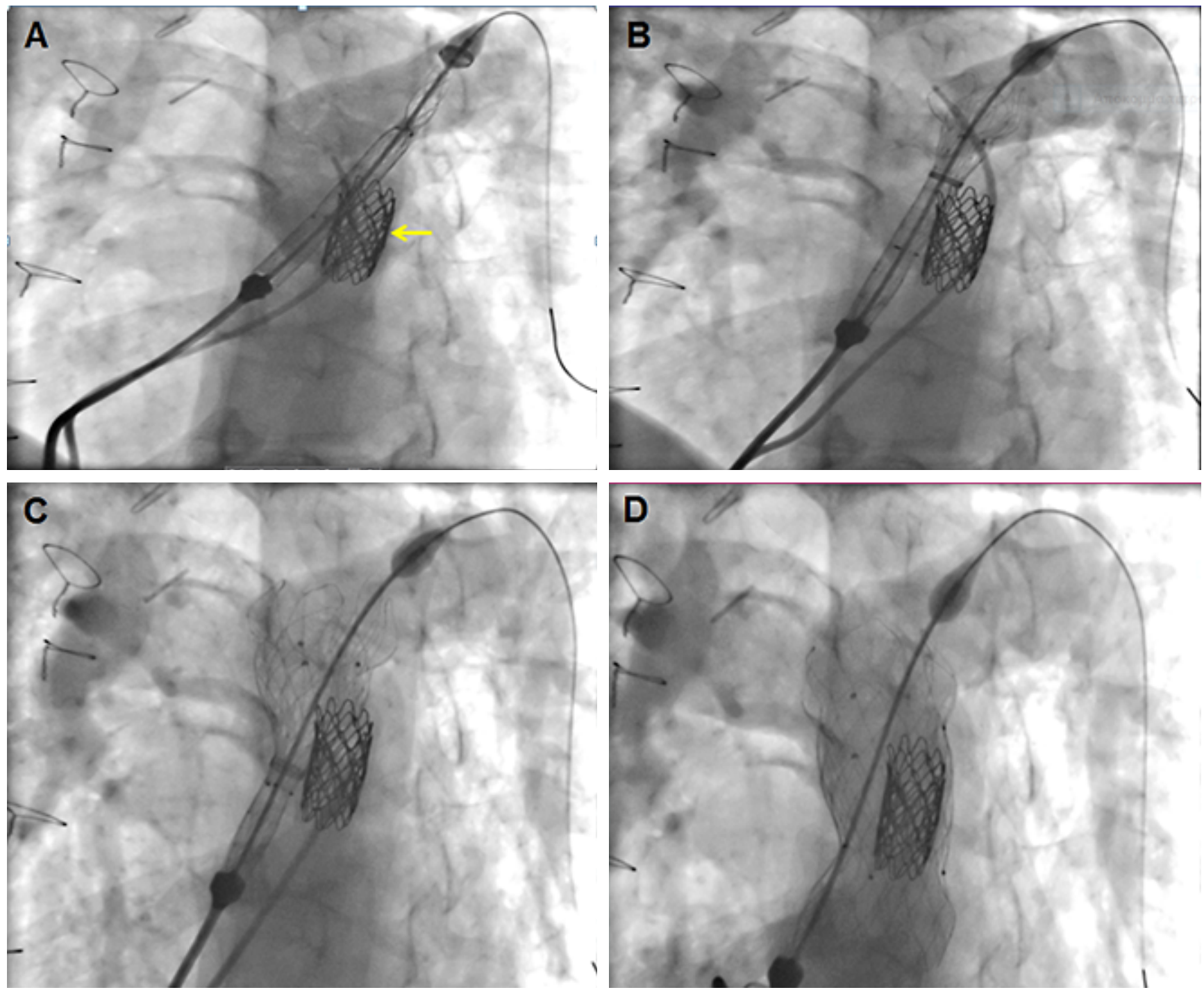

Figure 1. Panels $A, B, C, D$. Pulmonary angiograms in $L A O 40^{\circ}$ and cranial $15^{\circ}$ projections demonstrating the deployment steps of the Venus P-valve. (Panel A). Venus P-valve delivery system positioned across the right ventricular outflow tract (RVOT) with its tip in the proximal LPA. Deployment of the of the distal (Panel B) and central (Panel C) part of the valve. (Panel D). The valve is completed deployed with its proximal and distal flares expanded giving stability of the valve across the RVOT.

by partial resection of infundibular muscle and placement of a transannular patch. Nevertheless, the patient could not be weaned off bypass and therefore, a $14 \mathrm{~mm}$ Gore-Tex tube was placed between right ventricle (RV) and main pulmonary artery (MPA). The patient came off bypass and was discharged from the hospital in good clinical condition. During his follow-up the patient was doing quite well but over the years he developed progressively worsening exercise intolerance due to free pulmonary regurgitation through the Gore-Tex conduit that was confirmed by echocardiographic and cardiovascular magnetic Imaging (CMR) evaluation. The CMR estimated RVEDVi and RVEF were $170 \mathrm{ml} / \mathrm{m}^{2}$ and $45 \%$, respectively. Cardiac catheterization performed in 2008 showed a subpulmonary pressure gradient of $45 \mathrm{~mm} \mathrm{Hg}$ and mild pulmonary regurgitation across his native RVOT. In addition, a drop (from $125 \mathrm{~mm} \mathrm{Hg}$ to $90 \mathrm{~mm} \mathrm{Hg}$ ) of the aortic arterial pressure was observed during test balloon occlusion of the Gore-Tex tube indicating that the blood flow through native RVOT was not sufficient enough to maintain by itself a normal cardiac 
output. This could be attributed to the fact that the existed from the time of surgery residual infundibular stenosis had not yet completely regressed to permit the maintenance of a sufficient pulmonary blood flow exclusively though the native RNOT. After discussion with our surgeons and the family, in 2008, an 18 $\mathrm{mm}$ Melody ${ }^{\circledR}$ valve was placed successfully in the Gore-Tex tube. After the valve implantation the patient improved and he was doing quite well but 6 years later he developed again progressively aggravated exercise intolerance (NYHA class II -III symptoms) due to chronic pulmonary regurgitation this time through its operated native RVOT. In December 2017 echocardiographic and CMR evaluation revealed significant pulmonary regurgitation through the surgically placed transannular patch resulting in progressive dilatation of the RVOT and the pulmonary arteries. Doppler echocardiographic study showed a normal functioning Melody valve with no residual gradient and regurgitation. The CMR estimated RVEDVi and RVEF were $165 \mathrm{ml} / \mathrm{m}^{2}$ and $48 \%$, respectively. After the CMR study, the patient underwent cardiac catheterization with complete hemodynamic and angiographic studies. There was a $30 \mathrm{~mm} \mathrm{Hg}$ pressure gradient across the RVOT. The maximum systolic diameters of the proximal RVOT (just below the valve level), estimated valve annulus, proximal and distal MPA measured using biplane angiography, were 31 $\mathrm{mm}, 26 \mathrm{~mm}, 32 \mathrm{~mm}$ and $34.5 \mathrm{~mm}$, respectively. The length from the RVOT from the estimated valve annulus position to the PA bifurcation was $35 \mathrm{~mm}$. Balloon sizing of the pulmonary arteries was performed with maximal inflation of an ASD $34 \mathrm{~mm}$ Amplatzer sizing balloon simultaneously with an ascending aortogram that excluded coronary artery proximity and obstruction. Balloon sized RVOT/MPA diameters were only 1 $\mathrm{mm}$ larger than the angiographic ones. After obtaining procedural permission on compassionate basis from the Greek Federal Drug Administration and a written consent from the parents and the patient, it was decided to proceed to percutaneous pulmonary valve implantation with Venus P-valve.

To ensure stability and good opposition to the wall of the MPA, the Venus P-valve was selected to be $4 \mathrm{~mm}$ larger than the measured balloon waist at the pulmonary valve annulus level and equal to the maximal angiographic systolic diameter of the MPA.

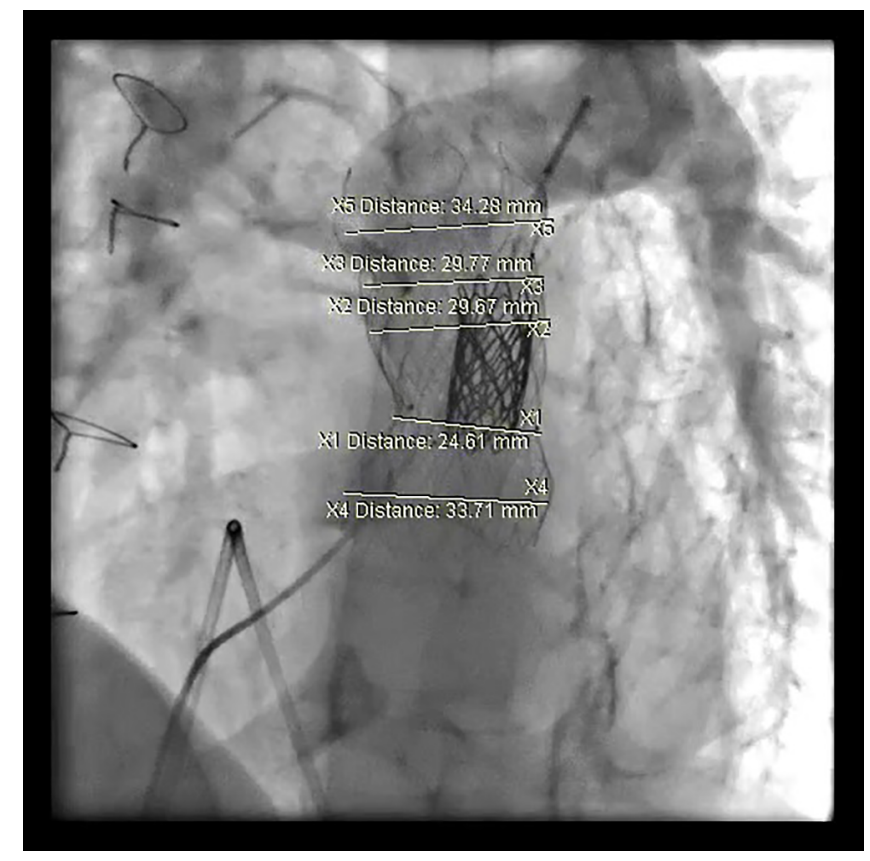

Video 1. Injection of contrast medium through a pig-tail 5F catheter into the proximal LPA showing competent Venus P-valve and Melody valves with no pulmonary regurgitation. View supplemental video at https://doi.org/10.12945/j.jshd.2019.024.18. sup.01.

The valve implantation was performed under general anesthesia using the previously described technique. [2-4] Based on the angiographic measurements and the anatomic characteristics of the RVOT, a $36 \mathrm{~mm}$ diameter and $35 \mathrm{~mm}$ length flared Venus P-valve was successfully implanted from the RVOT to MPA bifurcation through a $24 \mathrm{~F}$ delivery system. (Figure $1 \mathrm{~A}, \mathrm{~B}, \mathrm{C}, \mathrm{D}$ ). The procedure was guided using frequent check angiograms through a Berman angiographic catheter placed in the MPA/RV from a second femoral venous approach. Post procedural pulmonary angiography and hemodynamic measurements showed a well positioned and opposed to the wall of MPA competent valve and no pressure gradient across the RVOT (Video 1). The procedure and radiation times were 312 minutes and 48 minutes, respectively.

The patient was discharged the next day of the procedure on oral aspirin 3-5 mg/Kg/day for life. Before discharge an electrocardiogram (ECG) recording, a biplane chest X-ray, and a transthoracic echocardiography (TTE) were performed. Fluoroscopic examination 3 and six months after the procedure showed a good valve position with no fractures (Figure 3). At 

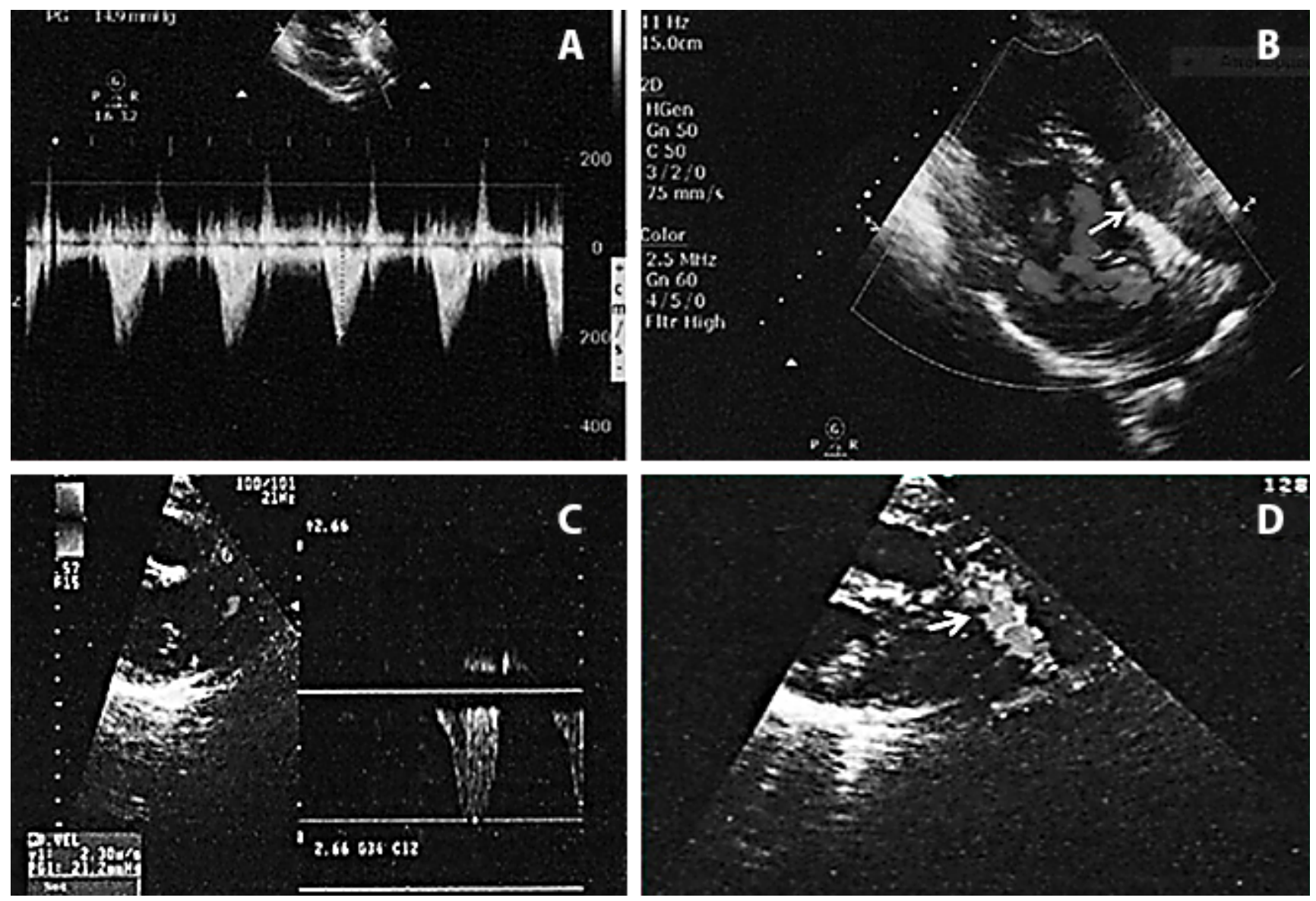

Figure 2. Panels $A, B, C, D$. Doppler and $2 D$ echocardiogram, respectively, obtained from right parasternal view (RVOT) at 1-year follow-up demonstrating competent Venus (Panels A, B) P-valve and Melody ${ }^{\circledR}$ (Panels C, D) valves with a 15 and $21 \mathrm{~mm}$ Hg peak pulmonary pressure gradient.

6 month and 1-year follow-up Doppler echocardiography showed a competent Venus P-valve with a peak pressure gradient of $15 \mathrm{~mm} \mathrm{Hg}$ across the RVOT (Figure $2 \mathrm{~A}, \mathrm{~B}$ ). Continued improvement of the RV indices was documented in CMR at 6 month (RVEDVi $-150 \mathrm{ml} / \mathrm{m}^{2}$, RVEF - 50\%) and 12 month (RVEDVi -120 $\left.\mathrm{ml} / \mathrm{m}^{2}, \mathrm{RVEF}-55 \%\right)$ follow-up. In addition, the patient reported significant progressive improvement in his clinical condition being in NYHA functional class I at 1 year follow-up. A TTE, an electrocardiogram and a chest- $X$ ray were scheduled to be performed at 1,6 , and 12 months after the procedure, and then serially once a year. It should be noted, that the patient and his parents were informed that following the implantation of Venous-P valve the Melody ${ }^{\circledR}$ valve is no longer needed and the obstruction of the Gore-text conduit using an occluding device should be contemplated.

\section{Discussion}

At the current time, the implantation of the two available CE approved pulmonary valves is mainly recommended in patients with dysfunctional RVOT conduits and patched RVOT with diameters up to 22 $\mathrm{mm}$ and to $26 \mathrm{~mm}$ for Melody and Edwards Sapien valves, respectively. Recently, the Edwards Sapien S3 valve has been implanted in native RVOTs with a maximal diameter of $29 \mathrm{~mm}$ with quite satisfactory short term results. However, these valves were not especially designed for use in patients with larger diameter native RVOTs with transannular patches. In addition, pre-stenting and stage implantation is required for their off-label use in native RVOT that increases the complexity of the procedure and the patient risk. ${ }^{1}$

The Venus P-valve is a recently introduced pulmonary valve designed for implantation in a wider range 
of post-operative patients with tetralogy of Fallot, who had transannular patch augmentation of RVOT. Recently, Cao et al [2], Promphan et al [3], and Garay et al [4] reported on their preliminary experience in humans using the Venus P-valve under Institutional approved protocols (the valve is not CE marked or FDA approved) with satisfactory early results. In this report a Melody and Venus P-valves were implanted sequentially in the rare case of a patient with post-operative TOF for the treatment of progressive dysfunction of an artificial and native RVOT, respectively. The procedures were successful with no complications and the patient was discharged the next day in excellent clinical condition. Fluoroscopy performed six months after the procedure, showed well-positioned valves with no fractures. Echocardiographic evaluation at the 1-year follow-up demonstrated a competent Venus P-valve with a small residual gradient across the RVOT. CMR follow-up showed significant improvement in RV volume and function parameters. Up to now approximately 110 Venous-P valves have been implanted worldwide with excellent short term results [5]. It should be noted that the Venous P- valve is being evaluated in CE trial for the assessment of its efficacy and safety.

\section{Conclusions}

Double PPVI can be used safely and effectively as an alternative to surgery for the treatment of selected post-operative patients with TOF and combined dysfunction of the native and surgically placed RVOT conduit. The Venus P-valve adds to our armamentar-

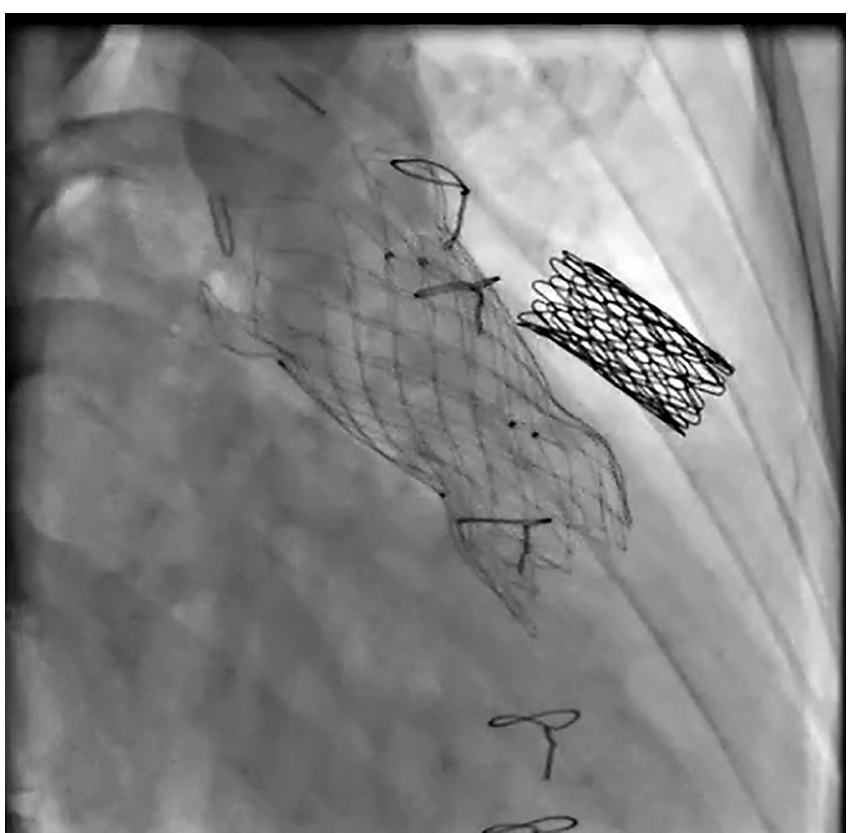

Figure 3. Fluoroscopic image six months after the Venus P-valve and Melody ${ }^{\circledR}$ valve implantation demonstrating absence of stent fractures.

ium for the treatment of patients with larger outflow tracts following surgical repair.

\section{Conflict of Interest}

The authors have no conflict of interest relevant to this publication.

\section{Comment on this Article or Ask a Question}

\section{References}

1. Asari MM, Cardoso R, Garcia D, Sandhu $S$, Horlick E, et al. Percutaneous pulmonary valve implantation. Present status and evolving feature. J Am Coll Cardiol. 2015;66:2246-2255. DOI: 10.1016/j. jacc.2015.09.055

2. Cao Q, Kenny D, Zhou D, Pan W, Guan L, Ge $J$, et al. Early clinical experience with a novel self-expanding percutaneous stent-valve in the native right ventricular outflow tract. Catheter Cardiovasc Interv. 2014;84:11311137. DOI: $10.1002 / \mathrm{ccd} .25544$

3. Promphan W, Prachasilchai P, Siripornpitak S, Qureshi SA, Layangool T. Percutaneous pulmonary valve implantation with the Venus P-valve: clinical experience and early results. Cardiol Young. 2015;26:698-710. DOI: $10.1017 /$ S1047951115001067

4. Garay F, Pan X, Zhang YJ, Wang C, Springmuller $D$. Early experience with the Venous $P$-valve for percutaneous pulmonary valve implantation in native pulmonary valve implantation in native outflow tract. Neth Heart J. 2017;25:76-81. DOI: 10.1007/ s12471-016-0932-5

5. AlkashkariW, Alrahimi J, Albugami S, Cao QL, Hijazi ZM. Transcatheter pulmonary valve replacement: The Venous-P valve current status. Structural Heart Disease. 2018;4:1-8. DOI: 10.12945/j.jshd.2018.035.17

Cite this article as: Thanopoulos $B D$, Dardas P, Kallifatidis A, Quireshi SA. Percutaneous Implantation of Venus P-Valve and Melody Valve in a Patient with Dysfunctional Native and Artificial Right Ventricular Outflow Tracts. Structural Heart Disease. 2019;5(1):1620. DOI: https://doi.org/10.12945/j. jshd.2019.024.18 